\title{
The Passivation Effect on Cd of Mixing of Amaranthus chinense L. Straw and Phosphate Rock Powder in Cd Polluted Soil
}

\author{
Xian Luo ${ }^{1}$, Zheng Zhao ${ }^{2}$, Fafu Deng ${ }^{2}$, Lin $\mathrm{Zhu}^{2}$, Haiyan Zhang ${ }^{2}$, Honglin $\mathrm{Pu}^{2}$, and Yongxia Jia ${ }^{2 *}$ \\ ${ }^{1}$ College of Horticulture, Sichuan Agricultural University, Chengdu, Sichuan, China \\ ${ }^{2}$ College of Resources, Sichuan Agricultural University, Chengdu, Sichuan, China
}

\begin{abstract}
Soil culture experiments were carried out to study the effects of the mixture of Amaranthus chinense L. straw and phosphate rock on soil $\mathrm{pH}$, available phosphorus (AP), available potassium (AK) and DTPA-Cd content in Cd polluted soil. The results showed that: (1) $2 \%$ phosphate rock powder combined with $1 \%, 2 \%, 3 \%$ of Amaranthus chinense L. straw can significantly increase soil $\mathrm{pH}$, AP and AK content. Compared with $\mathrm{CK}, \mathrm{pH}$ was increased by $0.42 \sim 0.56,0.53 \sim 0.70$ and $0.47 \sim 0.73$ units, respectively, and AP was increased by $19.03 \%$ 32.62\%, $14.91 \% \sim 33.63 \%$ and $18.88 \% \sim 58.01 \%$, respectively, and AK was increased by $201.46 \% \sim 234.66 \%, 210.5 \% \sim 266.56 \%$ and $184.07 \% \sim 224.98 \%$, respectively. (2) The DTPA-Cd content in soil decreased significantly after adding three different ratios of compound materials, which were $1.75 \%$ 9.01\%, 2.86\% 10.02\% and $0.98 \% \sim 8.7 \%$ lower than CK, respectively. In summary, the mixture of $1 \%$ Amaranthus chinense L. straw and $2 \%$ phosphate rock powder had the best passivation effect on $\mathrm{Cd}$.
\end{abstract}

\section{Introduction}

With the rapid development of modern industry and agriculture, the problem of cadmium (Cd) pollution in soil is becoming more and more serious [1,2]. According to the Chinese National Soil Pollution Survey Bulletin published in 2014, about 20 million hectare of cultivated land in China has been polluted by heavy metals to varying degrees, of which 33.33 million hectare is heavily and moderately polluted. The total over-standard rate of soil in China is $16.1 \%$. Cd is a harmful element in plant development. When $\mathrm{Cd}$ is absorbed and accumulated by plants beyond the critical concentration, plant growth will appear a series of toxic symptoms such as retardation, dwarfing, greening, yield and quality decline. Compared with other heavy metal elements, $\mathrm{Cd}$ is more active in the soil and easier to migrate to the human body through the food chain. Long-term consumption of Cd-contaminated rice will induce pain sickness and threaten human health. Therefore, the treatment of heavy metal $\mathrm{Cd}$ contaminated soil is urgent.

Using in situ passivation technology to reduce the bioavailability of $\mathrm{Cd}$ in soil is considered to be an effective and suitable treatment approach for China [3]. At present, there are many reports about using field crop straw to passivate $\mathrm{Cd}$ in soil. However, there are few reports about using $\mathrm{Cd}$ hyperaccumulator straw as passivator to remediate $\mathrm{Cd}$ polluted soil. Amaranthus chinense L. is a Cd hyperaccumulator [4], widely distributed in China. As an effective passivation material for remediation of heavy metal polluted soil, phosphate rock powder has the advantages of abundant resources and easy operation. Therefore, in this study, Amaranthus chinense L. straw and phosphate rock powder were used as materials to study the passivation of $\mathrm{Cd}$ in soil, in order to provide a theoretical reference for remediation of Cd polluted soil.

\section{Materials and Methods}

\subsection{Materials}

Phosphate rock powder: Phosphate rock powder was purchased from Sichuan Jinxin Mineral Products Co., Ltd., after grinding, pass through 100 mesh sieve.

The straw of Amaranthus chinense L. Straw was collected from Chongzhou Base of Sichuan Agricultural University and was not polluted by heavy metals. The plant straw was dried and pulverized with a micro-plant sample pulverizer (pore size of $1 \mathrm{~mm}$ ).

Cd polluted soil: Paddy soil was used in the experiment. After air-drying, the soil was ground to 5 $\mathrm{mm}$ sieve. The basic physical and chemical properties of Cd polluted soil are shown in Table 1.

\subsection{Experimental Design}

Using indoor culture experiments, $300 \mathrm{~g}$ of $\mathrm{Cd}$ polluted soil was weighed and placed in a $500 \mathrm{ml}$ plastic bottle. And adding different concentration of phosphate rock powder and Amaranthus chinense L. straw respectively

*Corresponding author: yongxiajia@163.com 
(Specific treatments is shown in table 2). At the same time, the control (CK) was set without adding any substance. And each treatment was repeated 3 times. Water was added to the culture bottle according to $65 \%$ of the field water holding capacity, sealed with plastic film, and left a few holes in the middle of the plastic film. The entire culture process was maintained at room temperature $\left(25{ }^{\circ} \mathrm{C}\right)$ and the soil moisture was maintained at about $65 \%$ of the field water holding capacity. After $0,5,15,30$ and 60 days of culture, soil samples were taken, air-dried and sieved, and various indicators were determined.

Table 1. The basic physical and chemical properties of $\mathrm{Cd}$ polluted soil. $\left(\mathrm{mg} \cdot \mathrm{kg}^{-1}\right)$

\begin{tabular}{ccccc}
\hline $\mathrm{pH}$ & $\mathrm{AP}$ & $\mathrm{AK}$ & $\mathrm{TN}$ & DTPA-Cd \\
\hline 6.88 & 50.99 & 150.81 & 758.84 & 3.71 \\
\hline
\end{tabular}

Table 2. Concentration setting of passivator for each treatment

\begin{tabular}{ccccc}
\hline & $\mathrm{CK}$ & $\mathrm{T} 1$ & $\mathrm{~T} 2$ & $\mathrm{~T} 3$ \\
\hline $\begin{array}{c}\text { straw } \\
\text { phosphate }\end{array}$ & 0 & $1 \%$ & $1 \%$ & $1 \%$ \\
rock powder & 0 & $1 \%$ & $2 \%$ & $3 \%$ \\
\hline
\end{tabular}

\subsection{Experimental Methods}

Soil $\mathrm{pH}$ was determined by potentiometry at a soil-liquid ratio of 1:2.5 [5]. Available phosphorus (AP) content in soil was determined by $\mathrm{NaHCO}_{3}$ extraction- $\mathrm{Mo}-\mathrm{Sb}$ colorimetry [5]. Available Potassium (AK) in soil was determined by $\mathrm{NH}_{4} \mathrm{OAC}$ Extraction-Flame Spectrophotometry [5]. Available $\mathrm{Cd}$ in soil was determined by DTPA solution Extraction-Atomic Absorption Spectrometry [5].

\subsection{Statistic Analyses}

Statistical analyses were performed using Microsoft Excel 2016 and SPSS 22.0 statistical software. Data were analyzed by one-way ANOVA with least significant difference (LSD) at 5\% confidence level.

\section{Results and Discussion}

\subsection{Effect of Compound Passivator on $\mathrm{pH}$ of $\mathrm{Cd}$ Polluted Soil}

As shown in Fig.1, with the extension of culture time, the soil $\mathrm{pH}$ increased after adding compound passivator. At the end of culture time $(60 \mathrm{~d}), \mathrm{T} 1, \mathrm{~T} 2$ and T3 treatment improved by $0.3,0.4$ and 0.48 units compared with $\mathrm{CK}$, respectively. In addition, the soil $\mathrm{pH}$ showed a trend of T3>T2>T1 throughout the culture process. Different proportions of phosphate rock powder and Amaranthus chinense L. straw can increase soil $\mathrm{pH}$, which is mainly because phosphate rock powder itself is alkaline. This is consistent with the results of $\mathrm{Xu}$ [6] and $\mathrm{Xu}$ [7].

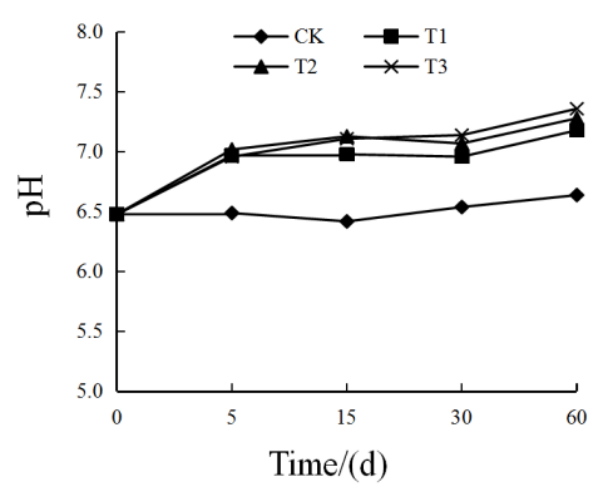

Fig. 1. $\mathrm{pH}$ in $\mathrm{Cd}$ polluted soil

\subsection{Effect of Compound Passivator on AP and AK of Cd Polluted Soil}

The content of AP and AK in Cd polluted soil increased significantly after adding compound passivator, and all periods showed the trend of T2> T3> T1 (Fig.2). The content of AP in soil tended to be stable at 15-30 days, and compared with CK, T1, T2 and T3 treatments increased by $20.5 \%-29.65 \%, \quad 40.85 \%-43.63 \%$ and $36.28 \%-39.31 \%$, respectively. AK content in soil tended to be stable at 5 days, at which time the three treatments increased by $215 \%, 210.5 \%$ and $225 \%$ compared with CK. The increase of AP and AK in soil is mainly due to the fact that Amaranthus chinense L. straw are used as an organic material, which increases the concent of $\mathrm{P}$ and $\mathrm{K}$ in the soil after decomposition. In addition, the decomposition of phosphate rock powder increases the AP content in the soil [8].
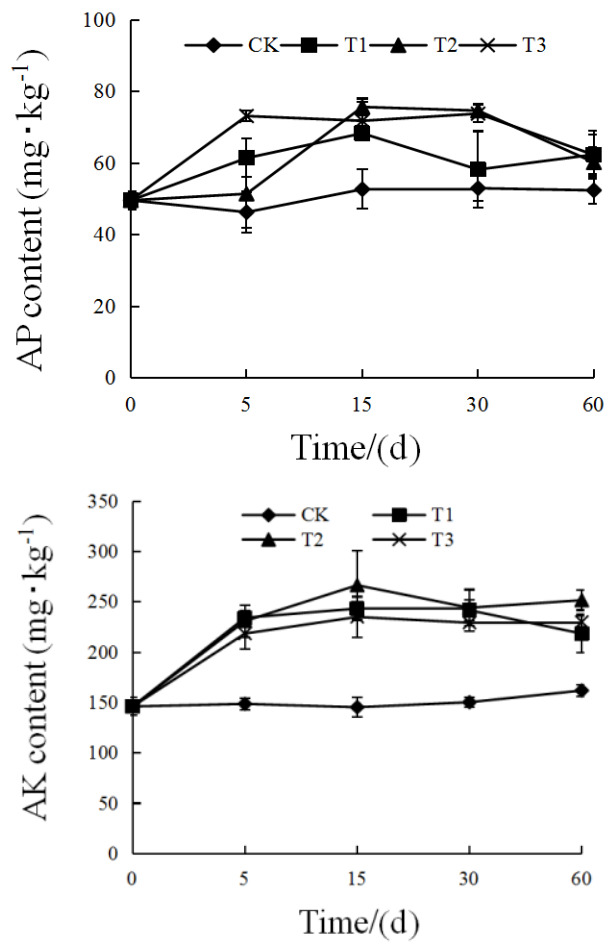

Fig. 2. AP and AK contents in Cd polluted soil 


\subsection{Effect of Compound Passivator on DTPA-Cd of Cd Polluted Soil}

As shown in Fig.3, after application of compound passivator to Cd polluted soil, the DTPA-Cd content in soil decreased significantly. At 30 days, the DTPA-Cd content tended to be stable, and compared with CK, the three treatments decreased by $9.01 \%, 9.39 \%$, and $6.86 \%$, respectively. In the later stage of culture, the DTPA-Cd content in soil showed a trend of $\mathrm{T} 2<\mathrm{T} 3<\mathrm{T} 1$. It indicates that $\mathrm{T} 2$ treatment has the best passivation DTPA-Cd in soil. The main reason is that the addition of alkaline phosphate rock powder increases the soil $\mathrm{pH}$, which can promote the increase of $\mathrm{Cd}$ exchange capacity on the soil surface, thereby reducing the DTPA-Cd content in the soil solution [9]. At the same time, the phosphate content in the soil increased, and $\mathrm{Cd}$ was stabilized by precipitation or direct adsorption of $\mathrm{Cd}$ on the surface of phosphate [10]. In addition, straw is added to the soil as an organic material, which contains a large number of organic functional groups that can form stable organic complexes with $\mathrm{Cd}$, thereby reducing the DTPA-Cd content in the soil [10].

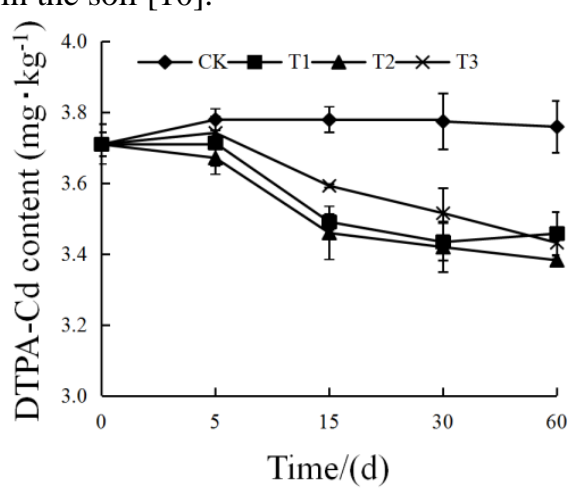

Fig.3. Effects of mixing of Amaranthus chinense L. straw and phosphate rock powder on available cd in cd-polluted soils

Adding alkaline inorganic materials and organic materials to heavy metal contaminated soils as composite passivators can improve soil $\mathrm{pH}$ to varying degrees. Previous study found that the soil $\mathrm{pH}$ increased by 1.5 and 0.7 units respectively after adding lime and organic fertilizer to $\mathrm{Cd}-\mathrm{Zn}$ contaminated loess. And the soil $\mathrm{pH}$ value increased significantly after adding active carbon and bentonite composite passivator to heavy metal contaminated soil. Similar to the above results, this study found that the $\mathrm{pH}$ value of $\mathrm{Cd}$-contaminated soil showed an upward trend with the increase of incubation time by adding grain amaranth straw and phosphate rock powder as organic-inorganic composite passivation materials. This is mainly due to the interaction between phosphate rock powder and soil solution, resulting in the release of phosphate and hydrogen phosphate anions, which ionize in soil solution, combine with hydrogen ions and displace hydroxyl groups on the surface of soil colloids; at the same time, phosphate rock powder can release alkaline metal ions such as $\mathrm{Ca}^{2+}, \mathrm{Mg}^{2+}$. Some of them can neutralize soil acidity, thereby increasing soil $\mathrm{pH}$. Further studies show that $\mathrm{pH}$ is the main factor affecting the bioavailability of heavy metals in soil. A series of reactions such as adsorption-desorption, precipitation dissolution of heavy metals in soil are strongly influenced by soil $\mathrm{pH}$. Therefore, the fixation of heavy metal ions is closely related to soil $\mathrm{pH}$. With the increase of soil $\mathrm{pH}$, all kinds of reactions occur. The adsorption performance of heavy metals on soil solid phase was improved, which reduced their bioavailability. It was found that the availability of heavy metal $\mathrm{Cd}$ in soil decreased significantly with the increase of soil $\mathrm{pH}$ after adding compound passivation materials. The main reason for the significant decrease of soil Cd bioavailability is that the addition of alkaline phosphate rock powder increases soil $\mathrm{pH}$ value, which can promote the increase of exchangeable adsorption of heavy metals on soil surface, thereby reducing the content of heavy metals in soil solution. At the same time, the content of phosphate in soil increases, because phosphate induces the adsorption of heavy metals. $\mathrm{Cd}$ is stabilized by precipitation with heavy metals or direct adsorption of heavy metals on phosphate surfaces.

Soil application of organic-inorganic passivation materials can improve basic fertility indicators such as available phosphorus and potassium. Previous study found that the available phosphorus in soil increased with the addition of phosphate rock powder and rice straw into lead-contaminated soil. Similar to this result, this study used $1 \%$ grain amaranth straw and 1\%,2\% and 3\% phosphate rock powder as passivation materials. It was found that the available phosphorus and potassium in soil increased rapidly with the increase of incubation time. Among them, the ratio of $1 \%$ grain amaranth straw and $2 \%$ phosphate rock powder had the best effect on the growth of available phosphorus and potassium in soil. This is mainly because the decomposition of phosphate rock powder increases the content of available phosphorus in soil. In addition, grain amaranth straw, as an organic material, increases the concentration of phosphorus and potassium in soil after decomposition. Meanwhile, with the increase of available phosphorus content in soil, available $\mathrm{Cd}$ showed a significant decreasing trend. People found that there was a significant negative correlation between the content of available $\mathrm{Cd}$ in soil and the amount of $\mathrm{Ca}, \mathrm{Mg}$ and $\mathrm{P}$ fertilizer added into the soil polluted by heavy metals. Similar to this conclusion, this experiment found that the content of available phosphorus in soil was related to the content of heavy metal $\mathrm{Cd}$ by adding organic and inorganic passivation materials to the soil polluted by heavy metals. There is a very significant negative correlation between effectiveness. The main reason for the significant decrease of available $\mathrm{Cd}$ content in soil is that on the one hand, phosphate ions in soil interact with $\mathrm{Cd}$ ions, so that heavy metal ions are fixed by precipitation and coordinated precipitation, thus reducing the effectiveness of $\mathrm{Cd}$. On the other hand, grain amaranth straw is added into the soil as organic matter, which contains a large number of organic functional groups which can form stable organic complexes with heavy metal ions, increase the cation exchange capacity in the soil, thereby reducing the available content of heavy metals in the soil. 


\section{Conclusion}

Adding the passivation material of Amaranthus chinense L. straw and phosphate rock powder to Cd- polluted soil can increase soil $\mathrm{pH}, \mathrm{AP}$ and $\mathrm{AK}$ content in different degrees, and significantly reduce the DTPA-Cd content in the soil. And 1\% Amaranthus chinense L. straw and 2\% of phosphate rock powder have the best effect.

\section{References:}

1. H. H. Wang, C. Wang, M. H. Zhang, Y. L. Pu, T. Li, Y. X. Jia. Acta Bot. Boreal.-Occident. Sin., 38: 2257-2266, (2018).

2. T. V. Dan, S. Krishnaraj, P. K. Saxena, Int. J. Phytoremediation, 2: 91-104, (2001).

3. F. Xie, C. H. Liang, Q. H. Meng, Y. D. Gao, S. Y. Song, Chinese J. Environ. Eng., 8: 3505-3510, (2014).

4. B. Song, Y. X. Zhang, M. L. Tian, Z. J. Yang, F. P. Wang, Chinese J. Environ. Eng., 13: 1711-1719, (2019).

5. S. D. Bao, Soil Agri. Chemi. Analysis. China Agri. Press, Beijing, (2000).

6. M. G. Xu, Q. Zhang, X. B. Zeng, Environ. Sci., 28: 1361-1366, (2007).

7. W. Q. Xu, S. Y. Sun, L. M. Hou, S. H. Ru, G. Y. Zhang, Acta Agriculterae Boreali-Sinica, 32 : 290-295, (2017).

8. W. G. Liu, Q. Zhao, Y. M. Yang, Chinese Agri. Sci. Bull., 34: 111-117, (2018).

9. S. W. Zhou, M. G. Xu, Acta Ecolo. Sinca, 27: 3043-3050, (2007).

10. S. Mishra, R. D. Tripathi, S. Srivastava, S. Dwivedi, P. K. Trivedi, O. P. Dhankher, A. Khare, Bioresource Tech., 100: 2155-2161, (2009). 